\title{
Static/dynamic input-based event-triggered control for a network resource constraint environment
}

This paper was downloaded from TechRxiv (https://www.techrxiv.org).

\section{LICENSE}

CC BY 4.0

SUBMISSION DATE / POSTED DATE

$21-12-2021 / 23-12-2021$

\section{CITATION}

sahu, poonam; Fulwani, Deepak (2021): Static/dynamic input-based event-triggered control for a network resource constraint environment. TechRxiv. Preprint. https://doi.org/10.36227/techrxiv.17312426.v1

DOI 


\title{
Static/dynamic input-based event-triggered control for a network resource constraint environment
}

\author{
Poonam Sahu, and Deepak M. Fulwani, Member, IEEE
}

\begin{abstract}
The work proposes static and dynamic input-based event-triggered controllers for a network resource-constrained environment. The controller is designed for a discrete-time system using a low-gain approach, where feedback gain is designed as a function of a user-defined parameter $\epsilon$. Depending on the event density, the low-gain parameter can be adjusted to increase the inter-event time between two consecutive events at a particular instant. Thus the demand for computational and network resources can be reduced. Finally a quarter car suspension system example is considered to illustrate the effectiveness of the proposed work.
\end{abstract}

Event-triggered control (ETC), Event-triggered mechanism (ETM), Low-gain feedback

\section{INTRODUCTION}

I $\mathrm{N}$ many control applications, the controller is implemented on a digital platform where the measurements are sampled periodically. The periodic sampling technique may lead to some redundant communication by over-utilizing the resources. However, in the network resource-constrained environment, it becomes imperative to adjust the resource requirements when resources are limited. This leads to the foundation of many theories, and one of the most significant works is event-triggered control (ETC). The seminal concept of ETC was developed in [1]-[3], and see the references therein. In ETC, scheduling of the control tasks is decided by an event in which the event is generated when the evolution of error involving state, output, or input of the system exceeds some pre-defined threshold value [2], [4]-[10]. This event-based aperiodic sampling mechanism makes ETC particularly more suitable under a resource-constrained environment. Further, many effective strategies, including static and dynamic ETM, are developed in [2], [4], [7], [11]-[22], [22], [23] and the references therein.

Most of the aforementioned ETMs are the function of system states or outputs, and the control update is decided based on the corresponding state/output-dependent ETMs. However, change in states may not always result in a significant change in input when compared with the input computed at the immediate previous instant. This happens because a linear control law $u(k)=G x(k)$ has states and a scaling factor contributed by some gain matrix $G$, where each parameter of $G$ may be either positive or negative. Assuming ndimensional system, $x(k)$ is represented by a column vector $\left[x_{1}, x_{2}, \ldots, x_{n}\right]^{T}$ and the gain matrix $G$ is represented by row vector $\left[G_{1}, G_{2}, \ldots, G_{n}\right]$. Suppose some gain vector parameters

P. Sahu and D. M. Fulwani are with the Electrical Department, Indian Institute of Technology Jodhpur, 342037, Rajasthan, India (e-mail: sahu.6@iitj.ac.in; e-mail:df@iitj.ac.in). are positive and some are negative. In that case, it is quite possible that the algebraic sum $G_{1} x_{1}+G_{2} x_{2}+\ldots+G_{n} x_{n}$ may lead to a very little change in control inputs irrespective of significant change in the system states. Thus, in this case, an update of the control law is not required. Hence, with statebased error, ETM causes redundant update of control law, which is further responsible for increasing events (and control updates) than needed. Furthermore, due to the increase in the number of events (control updates), communication costs and resource utilization are unnecessarily increased, which is not suitable for the resource constraint environment such as the networked control system due to increased network bandwidth. Please note that for some instances, actual control updates may also be more depending on how we chose the gain matrix and the corresponding state evolution, as discussed here. In the case of state/output-based ETM, it may also happen that control law changes significantly but still can't be updated if the corresponding state/output-based triggered condition is not satisfied. Therefore, with state/output-based error, ETM causes prolonged control task execution. Very few papers [6], [18], [24]-[26] have addressed the input-based ETC for different applications. In [24], the control law is computed asynchronously only at the sampling instant corresponding to the state event, therefore restricts the update of control law despite the input-based error considered in the ETM. In [6], [25], [26], the triggering mechanism is based on the input error where input is the function of estimated states. In [18], input-based ETM is proposed for the semi-global consensus of multi-agent systems where feedback gain is obtained by solving the algebraic Ricatti equation. Furthermore, in this work, we consider a mixed event-triggered threshold, which is a combination of a relative and an absolute threshold. A similar triggering threshold is studied in [24], [25], [27] where the triggering mechanism is based on the input error. In [24], the event-triggered threshold is a combination of an asymptotic threshold and absolute threshold. However, for a large value of time, the asymptotic threshold is zero, and then the triggering threshold is the same as the absolute threshold. Furthermore, the triggering threshold in [25], [27] consists of a relative threshold and an absolute threshold. However, the proposed ETM in this paper is different from the existing mixed triggering mechanism [24], [25], [27] such that the input error and control input can be dynamically adjusted to a new value depending on the network traffic.

Furthermore, most of the existing works on ETMs are static, which can cause redundant transmissions to an extent when controlled through a resource constrained network. To address the issue, in [7], a novel dynamic event-triggered mechanism 
(DETM) is proposed by introducing an auxiliary variable in the static ETM. It has been proved that the number of events in case of DETM is fewer than that of SETM. Motivated by [7], more related works on the DETM have been addressed in [22], [28]-[31]. Unlike the DETM considered in [7], [28], [29], another type of DETM is proposed in [32]-[35] by designing a dynamic variable according to an adaptive law, such that the triggering paradigm can dynamically adjust its value as per the system variations, including external disturbances, control traffic, etc. It should be noted that, events are triggered with a larger possibility under the dynamic ETM [32]-[34] and also results in a satisfactory control performance vis-a-vis the DETM proposed in [22], [28]-[31] and the static ETMs. This paper aims at the second type of DETM.

In many practical applications, while scheduling of control over networks, it requires longer inter-event times during heavy traffic on the network. The control input has a significant impact on inter-event times. Hence, in this paper, we use the seminal concept of the low gain feedback proposed by Lin et al. [36] for the design of the static and dynamic ETM, in which the control law is designed as a function of a small parameter $\epsilon$ such that the control magnitude goes to zero when $\epsilon$ approaches 0. Few works are reported in [11], [18], [21], [29], [37], [38], and the references therein, where this lowgain feedback is used with ETM to deal with the actuator failure, input saturation, output saturation, semi-global, and global stabilization, etc. for the discrete-time and continuoustime systems. Most of these ETMs with the low-gain feedback proposed in the literature [11], [18], [21], [29], [37], [38] are either state-dependent or output-dependent; as a result, control law may update without significant change in the input compared to the input computed at the immediately previous event.

\section{A. Main contribution}

1) A new input-based static ETM and dynamic ETM are proposed for a discrete-time system using a low-gain approach. The proposed ETMs update the control input when there is a significant change in control magnitude compared to the previous event.

2) In contrast to the work proposed in [29], in this paper, the low-gain variable $\epsilon(k)$ can be adjusted depending on the event density and network load. Further, it is analytically proved that the inter-event time at a particular instant is increased by decreasing the low-gain parameter. In contrast to the proposed static ETM, dynamic input has additional flexibility due to an introduction of dynamic variable in the triggered condition.

3) Ultimate boundedness of system states is established using ISS criteria. Further, asymptotic stability can be achieved by selecting design parameters appropriately.

4) To illustrate the effectiveness of the proposed ETM, we consider quarter-car active suspension control under a network resource constraint environment.

This paper is structured as follows. Section II introduces the preliminaries pertaining to the main results. Section III provides the main results of this paper. Section IV validates the effectiveness of the proposed theory through an illustrative

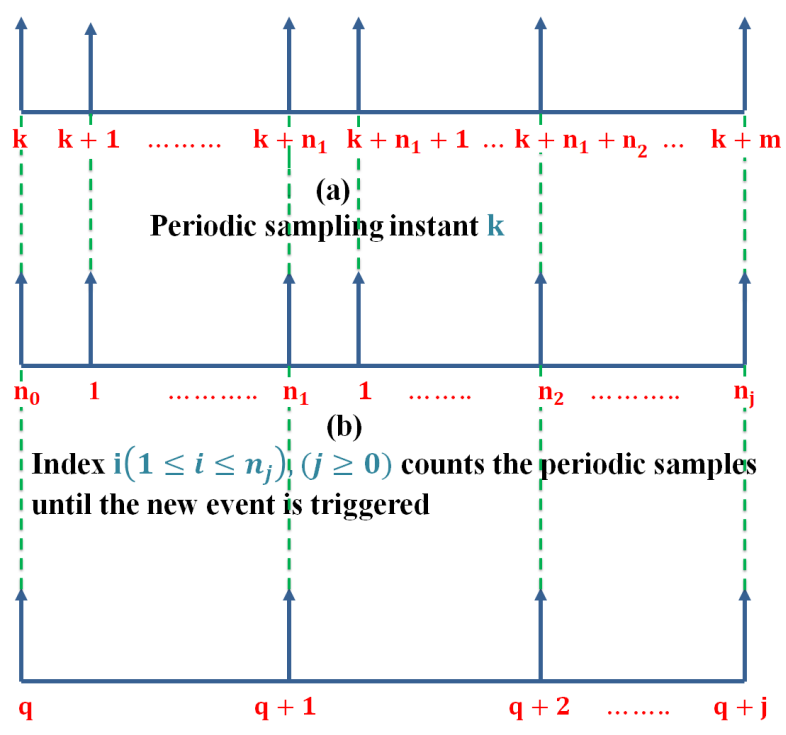

(c)

Index q corresponding to the event occurrence instant

Fig. 1.

example. In Section VI, conclusion is given along with a brief summary of the proposed work.

\section{B. Notation \& Definitions}

Index $k$ represents the sequence of periodic sampling instants. Events take place at some of these periodic instants at which event-triggered condition is satisfied. The instants at which events take place are denoted by $q, q+1, \ldots q+j$ respectively. Consider $n_{j},\left(n_{j} \in \mathbb{N}\right)$ number of periodic samples exist between two consecutive event-occurrence instants $q+j-1$ and $q+j, \forall j,(j \geq 1)$. Let us assume an event is triggered at $k^{\text {th }}$ instant and the next event is triggered at $\left(k+n_{1}\right)^{t h}$ instant and after this, the next event takes place at $\left(k+n_{1}+n_{2}\right)^{t h}$ i.e. $k, k+n_{1}, k+n_{1}+n_{2}, \ldots, k+n_{1}+\ldots+n_{j}$. To simplify the notation, we use index $q$ to denote these events i.e. $q+1=k+n_{1}, q+2=k+n_{1}+n_{2}$ and so on. Index $i$ in Fig. 1 counts the number of periodic samples between $(q+j-1)^{t h}$ and $(q+j)^{t h}$ event-occurrence instants, $\forall j,(j \geq 1) . j \in \mathbb{N}$ is a positive integer.

$T_{s}$ denotes the sampling period. A function $f:[0, a) \rightarrow$ $[0, \infty), a>0$ is said to be a class $K$ function, if $f$ is strictly increasing and $f(0)=0$. A function $f:[0, a) \rightarrow[0, \infty), a>$ 0 is said to be a class $K_{\infty}$ function if it belongs to class $K$, and $a \rightarrow \infty$. Also $f(m) \rightarrow \infty$ as $m \rightarrow \infty$.

Definition 1: [39] Consider system

$$
x(k+1)=A x(k)+B u(k)
$$

$A \in \mathbb{R}^{n \times n}$ and $B \in \mathbb{R}^{n \times m}$ denote the state matrix and input matrix respectively. A positive function $V(x(k))$ is said to be an ISS Lyapunov function if the following conditions are satisfied.

$$
\alpha_{1}(\|x(k)\|)<V(x(k))<\alpha_{2}(\|x(k)\|)
$$

$\alpha_{1}$ are $\alpha_{2}$ are $K_{\infty}$-function for all $x(k) \in \mathbb{R}^{n}$.

$$
V(x(k+1))-V(x(k)) \leq-\alpha_{3}(\|x(k)\|)+\zeta(\|u(k)\|)
$$




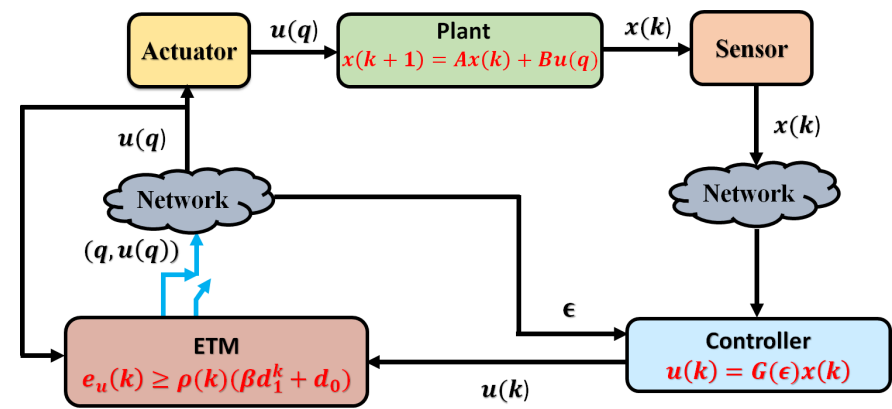

Fig. 2. Complete structure of the event-triggered closed-lop system where feedback loop of the system is closed over a communication network with it's respective plant, sensor, controller, ETM and actuator.

where the vectors $x(k) \in \mathbb{R}^{n}$ and $u(k) \in \mathbb{R}^{m}$ represent the state vector and input vector, respectively. $\alpha_{3}$ and $\zeta$ are the class $K_{\infty}$ and class $K$-function respectively.

\section{PRELIMinaries}

Consider that $(A, B)$ pair is stabilizable and eigenvalues of $A$ are located either inside or on the unit circle. We design a controller gain $G(\epsilon)$ as a function of a very small parameter $\epsilon$ using eigenstructure assignment [36] such that by reducing $\epsilon$, controller gain and control input are reduced.

The closed-loop eigenvalues $\forall \epsilon(0<\epsilon \leq 1)$ are obtained using the eigenstructure assignment [36]

$$
\lambda(A+B G(\epsilon))=(1-\epsilon) \lambda(A), \epsilon \in(0,1]
$$

$\lambda(A+B G(\epsilon))$ represents the closed-loop eigenvalues, which is a function of $\epsilon$ and control input is defined as

$$
u(k)=G(\epsilon) x(k)
$$

System with the low-gain feedback $G(\epsilon), \forall \epsilon \in(0,1]$, satisfies the following properties [36].

$$
\begin{aligned}
& \text { Lemma 1: [36] }\|G(\epsilon)\| \leq \psi \epsilon, \psi>0 \\
& \left\|(A+B G(\epsilon))^{k}\right\| \leq \frac{\psi_{1}}{\epsilon^{m-1}}(1-\epsilon)^{\frac{k}{2}}, \psi_{1}>1, \forall k \geq 0
\end{aligned}
$$

$m$ denotes the largest algebraic multiplicity of the eigenvalues of $A$.

$$
\left\|G(\epsilon)(A+B G(\epsilon))^{k+r}\right\| \leq \Gamma \epsilon(1-\epsilon)^{\frac{k}{2}}, \Gamma>0, \forall k \geq 0
$$

$r$ represents the number of open loop eigenvalues located inside the unit circle. $\psi, \psi_{1}$, and $\Gamma$ are constant positive scalars, which are independent of $\epsilon$.

Remark 1: Property (6) shows the asymptotic behavior of the control input (5) i.e. $\lim _{\epsilon \rightarrow 0}\|G(\epsilon)\|=0$. Property (7) indicates the slow peaking phenomena of the system states (1) under the feedback gain $G(\epsilon)$ while property (8) shows that $L_{\infty}$ norm of the control input $u(k)$ and all its derivatives are reduced by reducing $\epsilon$ for any given initial conditions.

\section{MAIN RESUlts}

The first part of this section proposed event-triggered rule for a network resource-constrained environment. The second part of this section proves the stability of the system while the third part establishes the relation between the inter-event time and $\epsilon$. Before proceeding to the system stability, the closedloop system with the event-triggered input $u(q)$ is obtained as follows.

\section{A. Event-triggered mechanism}

In this section, we introduce the improved dynamic ETM. The main objective of the proposed dynamic ETM is to avoid the redundant update of the control law using input-based error in the triggering mechanism and also to control the event density dynamically depending on error dynamics and event density.

Consider the event is triggered at $k=(q+j-1)^{t h}$ instant, and the next event is triggered at $k+n_{j}=(q+j)^{t h}$ instant as shown in the Fig. 1, the error at $k^{t h}$ instant and $\left(k+n_{j}\right)^{t h}$ instant are equal to zero, where $n_{j}$ is a positive integer and $j=\{1,2, \ldots\}$. Error at the sampling instances between two events is given by

$$
e_{u}(k+i-1):=u(k)-u(k+i-1), 1<i \leq n_{j}
$$

Consider the current event is triggered at $k=(q+j-1)^{t h}$ instant, the next event is generated if the following dynamic triggered condition is satisfied.

$$
q+j=\inf \left\{k>q+j-1,\left\|e_{u}(k)\right\|>\sigma(k)\left(d_{0}+\beta d_{1}^{k}\right)\right\}
$$

$k$ in (10) represents the sequence of periodic sampling instants i.e. $k \in[q+j-1, q+j]$ at which event-triggered condition is to be checked. $q+j$ represents the sequence of triggering instances $\{q+1, q+2, \ldots\},, \forall j \geq 1 . d_{1}, d_{0}\left(0<d_{1}, d_{0}<\right.$ $1), \beta>0$ are the design parameters. $\sigma(k)$ is a dynamic variable, which can be computed as

$$
\sigma(k+1)=\left(\bar{\theta} c_{1}^{k}+\frac{\theta\left(c_{3}^{k}\right)}{1+\left\|e_{u}(k)\right\|}\right) \sigma(k)+c_{2}
$$

$c_{1},\left(0<c_{1}<1\right)$ is an arbitrary scalar with an initial condition $\sigma(0) \geq 0,0<\bar{\theta}<1 . c_{3}$ is chosen such that it satisfies $c_{3}<\min \left\{c_{1}, c_{1} \frac{\left\|e_{u}(k)\right\|}{\left\|e_{u}(k-1)\right\|}\right\}$ and $\theta$ is selected such that $\theta<1-\bar{\theta} . c_{2}$ is an arbitrary scalar whose value is selected such that $c_{2} \leq\left(1-\bar{\theta}-\frac{\theta}{1+\left\|e_{u}(0)\right\|}\right) \sigma(0)$ is satisfied. The DETM proposed in (10) has both the asymptotic threshold and absolute threshold terms; hence it can avail the benefits of both the triggering mechanisms [40].

Remark 2: Unlike the existing DETMs [22], [29], [30], the proposed DETM provides better system performance while reducing the network load.

Remark 3: It should be noted that both the conventional time-triggering paradigm and static paradigm are considered as the two special cases of the DETM proposed in (10). The developed DETM (10) coincides with the conventional periodic scheme for $c_{1}=1, \sigma(0)=0$ and $c_{2}=0$. The proposed ETM coincides with the static triggering scheme when $c_{1}=1, c_{3}=0$ and $\sigma(0) \geq 0$.

Remark 4: The proposed ETM in (10) is triggered with a larger possibility due to the presence of dynamic variable $\sigma(k)$, and design parameters $d_{0}, d_{1}$ but results in a better network performance vis-a-vis dynamic ETMs proposed in [22], [30], [31]. Unlike the static ETMs [21], [41], the proposed static input-based ETM (for the given case when $c_{1}=1, c_{3}=0$, and $\sigma(0) \geq 0$ ) can save more network resources by avoiding redundant control updates. Specifically, if an event occurs at $q^{t h}$ instant, the next event occurrence instant can be made 
larger by decreasing the low-gain parameter at that particular instant during high control traffic or event density.

Lemma 2: Consider the proposed DETM (10) and dynamic rule (11). For the given dynamic variable $\sigma(k)$ with an initial condition $\sigma(0) \in(0,1]$, the following inequality holds.

$$
0<\ldots \leq \sigma(k+1) \leq \sigma(k) \leq \sigma(0) \leq 1, \forall k \in \mathbb{N}
$$

Furthermore, $\sigma(k)$ is a monotone decreasing and bounded sequence.

Proof: The proof of the above Lemma can be performed by using the mathematical induction method. As by design $\sigma(0) \in$ $(0,1]$; therefore, it is straightforward that (12) will hold for $\left.\sigma(k)\right|_{k=0}$. Now substituting $k=0$ in (11), $\sigma(1)$ is obtained as

$$
\sigma(1)=\left(\bar{\theta}+\frac{\theta}{1+\left\|e_{u}(0)\right\|}\right) \sigma(0)+c_{2}
$$

Since by design $\bar{\theta}+\frac{\theta}{1+\left\|e_{u}(0)\right\|}<1$ and $c_{2} \leq(1-\bar{\theta}-$ $\left.\frac{\theta}{1+\left\|e_{u}(0)\right\|}\right) \sigma(0)$; therefore $\sigma(1) \leq \sigma(0) \leq 1$. Similarly substituting $k=1$ in (11), we obtain

$$
\sigma(2)=\left(\bar{\theta} c_{1}^{1}+\frac{\theta\left(c_{3}\right)}{1+\left\|e_{u}(1)\right\|}\right) \sigma(1)+c_{2}
$$

Noticing that $\left(\bar{\theta} c_{1}^{1}+\frac{\theta\left(c_{3}\right)}{1+\left\|e_{u}(1)\right\|}\right)<1$ and $c_{2}$ is a fixed scalar, therefore; subtracting $\sigma(1)$ from $\sigma(2)$, we have

$$
\begin{aligned}
& \sigma(2)-\sigma(1)= \\
& \left(\bar{\theta} c_{1}^{1}+\frac{\theta\left(c_{3}\right)}{1+\left\|e_{u}(1)\right\|}\right) \sigma(1)-\left(\bar{\theta}+\frac{\theta}{1+\left\|e_{u}(0)\right\|}\right) \sigma(0) \\
& \sigma(2)-\sigma(1)=\bar{\theta}\left(c_{1} \sigma(1)-\sigma(0)\right)+ \\
& \frac{\theta\left(c_{3} \sigma(1)-\sigma(0)\right)+\theta\left(\left(c_{3}\right) \sigma(1)\left\|e_{u}(0)\right\|-\sigma(0)\left\|e_{u}(1)\right\|\right)}{\left(1+\left\|e_{u}(1)\right\|\right)\left(1+\left\|e_{u}(0)\right\|\right)}
\end{aligned}
$$

As by design $\bar{\theta}<1, c_{1}<\min \left\{1, \frac{\sigma(k-1)}{\sigma(k)}\right\}, c_{3}<$ $\min \left\{c_{1}, c_{1} \frac{\left\|e_{u}(k-1)\right\|}{\left\|e_{u}(k)\right\|}\right\}$. For the given values of parameters $c_{1}, c_{3},(\sigma(2)-\sigma(1))<0$, which implies $\sigma(2) \leq \sigma(1)$ Following the same procedure, it can be obtained that

$$
\sigma(k+1) \leq \sigma(k) \leq \sigma(0)<1, \forall k \in \mathbb{N}
$$

which implies that the dynamic variable $\sigma(k), \forall k \in \mathbb{N}$ is a monotonically decreasing sequence. Using (11) and (17), we can obtain

$$
\begin{gathered}
\sigma(k) \geq \sigma(k+1)=\left(\bar{\theta} c_{1}^{k}+\frac{\theta\left(c_{3}^{k}\right)}{1+\left\|e_{u}(k)\right\|}\right) \sigma(k)+c_{2} \\
\sigma(k)\left(1-\bar{\theta} c_{1}^{k}-\frac{\theta\left(c_{3}^{k}\right)}{1+\left\|e_{u}(k)\right\|}\right) \geq c_{2} \\
\sigma(k) \geq \frac{c_{2}}{\left(1-\bar{\theta} c_{1}^{k}-\frac{\theta\left(c_{3}^{k}\right)}{1+\left\|e_{u}(k)\right\|}\right)}
\end{gathered}
$$

As $k \rightarrow \infty, \bar{\theta} c_{1}^{k}=0, c_{3}^{k}=0$, which results in $\sigma(k) \geq c_{2}$. Assuming an arbitrary small positive constant $\bar{\sigma}$ such that $\sigma(k)$ can be further lower bounded as

$$
\sigma(k) \geq \bar{\sigma}, \forall k \in \mathbb{N}
$$

where, $\bar{\sigma}=c_{2}$. However, $\sigma(k)$ is asymptotically converging for $c_{2}=0$, which stisfies the inequality (12). This completes the proof.

\section{B. Event-triggered closed loop system \& stability}

Before establishing system stability, we first present eventtriggered closed-loop system and a Lemma, which are used in the subsequent Theorem.

Fig. 2 shows the complete structure of the Event-triggered closed loop system whose feedback loop is connected over a communication network. It consists of a plant, sensors, controller, ETM, and actuator. The controller receives the state information from the sensor through a network and then calculates the control input. At each sampling instant, ETM decides whether a control law should be updated and sent to the actuator through a network or not. However, the decision of the ETM is based on the triggering condition proposed in (10). When an event is triggered, ETM sends the updated control input to the actuator through the network. If the event is not triggered, then the actuator uses the past updated information of the control law and sends it to the plant.

Consider the event is triggered at $k=(q+j-1)^{t h}, j \geq 1$ instant, event-triggered input is given by

$$
u(k)=u(q+j-1), q+j-1 \leq k<q+j
$$

where, $(q+j)^{t h}$ instant represents the next event-occurrence instant. Now using (22), the system (1) at $(k+i)^{t h}, 1 \leq i \leq n_{j}$ instant is given by

$$
x(k+i)=A x(k+i-1)+B u(k)
$$

Using (9), the system represented in (23) is further rewritten as

$$
\begin{aligned}
& x(k+i)=A x(k+i-1)+B u(k+i-1)+B e(k+i-1) \\
& x(k+i)=(A+B G(\epsilon)) x(k+i-1)+B e(k+i-1)
\end{aligned}
$$

To simplify the notation, define $k+i:=s+1$. Substituting $k+i$ by $s+1, x(k+i)$ in (25) is written as

$$
\begin{aligned}
& x(s+1)=(A+B G(\epsilon)) x(s)+B e_{u}(s) \\
& x(s+1)=A_{d} x(s)+B e_{u}(s)
\end{aligned}
$$

where, $A_{d}:=A+B G(\epsilon)$.

Lemma 3: For any two vectors $M_{1} \in \mathbb{R}^{n}, M_{2} \in \mathbb{R}^{n}$ and a scalar $\phi>0$, the following relation holds.

$$
2\left(M_{1}\right)\left(M_{2}\right) \leq \phi\left(M_{1}^{T} M_{1}\right)+\frac{1}{\phi}\left(M_{2}^{T} M_{2}\right)
$$

Now considering $M_{1}=x(s)$, and $M_{2}=A_{d}^{T} P B e_{u}(s)$, we can write $2 x^{T}(s) A_{d}^{T} P B e_{u}(s)$ as

$$
\begin{aligned}
2 x^{T}(s) & A_{d}^{T} P B e_{u}(s) \leq \phi\left(x^{T}(s) x(s)\right) \\
& +\frac{1}{\phi} e_{u}^{T}(s)\left(A_{d}^{T} P B\right)^{T}\left(A_{d}^{T} P B\right) e_{u}(s)
\end{aligned}
$$

Theorem 1: Consider the triggering rule (10), and the feedback gain $G(\epsilon)$ designed as per (4), the state trajectory $x(s)$ 
of the event-triggered closed loop system (26b) is uniformly ultimate bounded with center at origin and radius

$$
r:=d_{0} \sqrt{\frac{\delta_{e} c_{2}}{\delta_{x}}}+\bar{\Delta}
$$

where, $\bar{\Delta}$ is a small arbitrary positive constant.

$$
\begin{aligned}
\delta_{x} & :=\lambda_{\min }(Q)-\phi \\
\delta_{e} & :=\left(\left\|B^{T} P B\right\|+\frac{\left\|A_{d}^{T} P B\right\|^{2}}{\phi}\right)
\end{aligned}
$$

where, $\phi>0$ is selected such that the following holds.

$$
\phi<\lambda_{\min }(Q)
$$

Proof: As $A_{d}$ is Schur stable by design, for the given $Q>0$, there exists a positive definite matrix $P>0$ which is the unique solution of the following Ricatti equation

$$
A_{d}^{T} P A_{d}-P=-Q
$$

Consider an ISS Lyapunov candidate function $V(x(s))$ for system (26b) as

$$
V(x(s), \sigma(s))=x(s)^{T} P x(s)+\sigma(s)
$$

which satisfies the following inequality

$$
\lambda_{\min }(P)\|x(s)\|^{2} \leq x(s)^{T} P x(s) \leq \lambda_{\max }(P)\|x(s)\|^{2}
$$

where, $\lambda_{\min }(P)$ and $\lambda_{\max }(P)$ denote the minimum eigenvalue and maximum eigenvalue of $P$. Using (26b), the forward difference of $V$ is computed as

$$
\Delta V=V(x(s+1), \sigma(s+1))-V(x(s), \sigma(s))
$$

Using (33), $\Delta V$ in (35) can be computed as

$$
\begin{aligned}
\Delta V & =x^{T}(s)\left(A_{d}^{T} P A_{d}-P\right) x(s)+e_{u}^{T}(s) B^{T} P A_{d} x(s) \\
& +x^{T}(s) A_{d}^{T} P B e_{u}(s)+e_{u}^{T}(s) B^{T} P B e_{u}(s) \\
& +\sigma(s+1)-\sigma(s)
\end{aligned}
$$

$\Delta V$ in (36) is further simplified by using (32) as

$$
\begin{gathered}
\Delta V=x^{T}(s)(-Q) x(s)+2 x^{T}(s) A_{d}^{T} P B e_{u}(s) \\
+e_{u}(s)^{T} B^{T} P B e_{u}(s)+\sigma(s+1)-\sigma(s)
\end{gathered}
$$

Using Lemma 3, $\Delta V$ in (37) is bounded by using (11), (34), and (28) as

$$
\begin{aligned}
& \Delta V \leq\left(-\lambda_{\min }(Q)+\phi\right)\|x(s)\|^{2} \\
& +\left(\left\|B^{T} P B\right\|+\frac{\left\|A_{d}^{T} P B\right\|^{2}}{\phi}\right)\left\|e_{u}(s)\right\|^{2} \\
& +\left(\bar{\theta} c_{1}^{s}+\frac{\theta\left(c_{3}^{s}\right)}{1+\left\|e_{u}(s)\right\|}\right) \sigma(s)+c_{2}-\sigma(s)
\end{aligned}
$$

Using (10), $\Delta V$ in (38) is bounded by

$$
\begin{aligned}
& \Delta V \leq\left(-\lambda_{\min }(Q)+\phi\right)\|x(s)\|^{2} \\
& +\left(\left\|B^{T} P B\right\|+\frac{\left\|A_{d}^{T} P B\right\|^{2}}{\phi}\right)\left(\sigma^{2}(s)\left(d_{0}+\beta d_{1}^{s}\right)^{2}\right) \\
& +\left(\bar{\theta} c_{1}^{s}+\frac{\theta\left(c_{3}^{s}\right)}{1+\left\|e_{u}(s)\right\|}\right) \sigma(s)+c_{2}-\sigma(s)
\end{aligned}
$$

Using (30a), (30b), the above inequality (39) is reduced to

$$
\begin{aligned}
& \Delta V \leq-\delta_{x}\|x(s)\|^{2} \\
& +\left(\delta_{e} d_{0}^{2} \sigma(s)+\left(\bar{\theta} c_{1}^{s}+\frac{\theta\left(c_{3}^{s}\right)}{1+\left\|e_{u}(s)\right\|}\right)-1\right) \sigma(s) \\
& +\delta_{e} \beta^{2}\left(d_{1}^{s}\right)^{2}+c_{2}
\end{aligned}
$$

Now using (21), $\Delta V$ can be further bounded as

$$
\begin{aligned}
& \Delta V \leq-\delta_{x}\|x(s)\|^{2} \\
& +\left(\frac{\delta_{e} d_{0}^{2} c_{2}}{1-\bar{\theta} c_{1}^{k}-\frac{\theta c_{3}^{k}}{1+\left\|e_{u}(k)\right\|}}+\bar{\theta} c_{1}^{s}+\frac{\theta\left(c_{3}^{s}\right)}{1+\left\|e_{u}(s)\right\|}-1\right) \\
& \left(\frac{c_{2}}{1-\bar{\theta} c_{1}^{k}-\frac{\theta c_{3}^{k}}{1+\left\|e_{u}(k)\right\|}}\right)+\delta_{e} \beta^{2}\left(d_{1}^{s}\right)^{2}+c_{2}
\end{aligned}
$$

As $s \rightarrow \infty, d_{1}^{s}=0, c_{1}^{s}=0, c_{3}^{s}=0$. Therefore,

$$
\Delta V \leq-\delta_{x}\|x(s)\|^{2}+\left(\delta_{e} d_{0}^{2} c_{2}-1\right) c_{2}+c_{2}
$$

which further simplified into

$$
\Delta V \leq-\delta_{x}\|x(s)\|^{2}+\delta_{e} d_{0}^{2} c_{2}
$$

$\Delta V<0$ if $\delta_{x}\|x(s)\|^{2}>\delta_{e} d_{0}^{2} c_{2}$, which further results in

$$
\|x(s)\|>d_{0} \sqrt{\frac{\delta_{e} c_{2}}{\delta_{x}}}
$$

It can be observed from (44) that the state trajectory will enter in an ellipsoid which has center at origin and has radius $r:=d_{0} \sqrt{\frac{\delta_{e} c_{2}}{\delta_{x}}}+\bar{\Delta}, \bar{\Delta}$ is an arbitrary positive constant. This completes the proof.

Corollary 1: For $d_{0}=0, d_{1} \neq 0$, state trajectories of the closed-loop event-triggered system (26b) will asymptotically converge to the origin.

Corollary 2: For static ETM, the closed-loop system stability can be proved using the same procedure as in Theorem 1 by considering $c_{1}=1, c_{3}=0$. It should be noted that, for $c_{1}=1, c_{3}=0$, the dynamic variable $\sigma(s)=\bar{\theta} \sigma(0)+c_{2}>$ $0, \forall s \geq 0$. Therefore, we consider Lyapunov candidate function $V(x(s))$ as $x^{T}(s) P x(s)$. Following the same procedure as given in Theorem 1, we obtain $\Delta V<0$ for

$$
\|x(s)\|>\left(\bar{\theta} \sigma(0)+c_{2}\right) d_{0} \sqrt{\frac{\delta_{e}}{\delta_{x}}}
$$

which implies system trajectories enters into an ellipsoid with center at origin and has radius equal to $\left(\bar{\theta} \sigma(0)+c_{2}\right) d_{0} \sqrt{\frac{\delta_{e}}{\delta_{x}}}$. $\delta_{e}, \delta_{x}$ are defined in (30a) and (30b), respectively.

\section{How to adjust $\epsilon$ according to network traffic}

In this section, we consider NCSs, where the controller sends the updated control input to the actuator through a network. We use event-triggered conditions from controller to actuator network to avoid redundant control updates and to reduce control traffic. In this paper, the control traffic is measured by the event density (i.e., number of control updates in the given run time). Based on the event-triggered closedloop system, here we propose Algorithm to calculate the control traffic (or event density) for the given period. 
To measure the traffic and to detect the events, we construct the variables $R(k)$ and $N(k)$ as

$$
\begin{gathered}
R(k)= \begin{cases}1, & \text { if control law is updated } \\
0, & \text { otherwise }\end{cases} \\
N(k)=\frac{1}{n}\left[\sum_{l=0}^{l=k} R(k)\right]
\end{gathered}
$$

$n$ denotes the number of periodic samples for the given run time. For convenience, the control traffic logic can be considered as

$$
\begin{cases}N(k) \leq N^{+}, & \text {if control traffic is not caused } \\ N(k)>N^{+}, & \text {if control traffic is caused }\end{cases}
$$

where, $\mathrm{N}^{+}$is the user-defined variable, which represents the upper bound of $N(k)$. To bring further clarity in the process of the adaptively adjusted low-gain variable as per the network traffic ( control traffic) condition, here in the following, we introduce the algorithm.

Algorithm:

1) Set the appropriate values of triggering parameters $d_{0}, d_{1}$, and initial sampling instant $k=k_{0}$. Initialize the low-gain variable $\epsilon(k)=\epsilon\left(k_{0}\right)$ and dynamic variable $\sigma(k)=\sigma\left(k_{0}\right)$. Calculate the value of $N\left(k_{0}\right)$. Introduce an array, which records the sampling instants at which ETC (10) is satisfied. Choose the run time " $t$ ". Consider $N_{u t}$ and $N_{t}$ as the number of continuously untriggered and triggered events, respectively. Set $N_{u t}^{+}$and $N_{t}^{+}$as the upper bounds of $N_{u t}$ and $N_{t}$, respectively. Introduce $R_{u t}=0$ and $R_{t}=0$ as the counters . Set appropriate minimum and maximum values of low-gain variable as $\epsilon_{m}, \epsilon_{M}$, respectively, where $0<\epsilon_{m}, \epsilon_{M}<1$. Chose the initial low-gain variable $\epsilon\left(k_{0}\right)$ such that it satisfies $\epsilon_{m}<\epsilon\left(k_{0}\right)<\epsilon_{M}$.

2) If the event is detected and control law is updated then go to step 4. If the event is not detected and actuator receives past value of control signal through network then go to step 3 .

3) If traffic possibility is higher than the given threshold, then go to step 5, else go to step 6 .

4) Set $k=k+1$ and update the values of $N_{t}=N_{t}+$ $1, N_{u t}=0, R_{t}=R_{t}+1, R_{t}=R_{t}+1$, if when event is not detected. If the control traffic is high, i.e. if $N_{t} \leq$ $N_{t}^{+}$, go to step 6. If $N_{t}>N_{t}^{+}$and $\epsilon(k)>\epsilon_{m}$, then set $N_{t}=0, R_{t}=R_{t}+1$ and $\epsilon(k)=\epsilon(k) * 0.5$ and go to step 6. If $N_{t}>N_{t}^{+}$and $\epsilon(k) \leq \epsilon_{m}$, then keep $\epsilon(k)$ unaltered and go to step 6 .

5) Increase the values $N_{u t}=N_{u t}+1$ and $R_{u t}=R_{u t}+1$. If the control traffic is high i.e. $N_{u t} \neq N_{u t}^{+}$, go to step 6. If $N_{u t}>N_{u t}^{+}$and $\epsilon(k)>\epsilon_{m}$, then set $N_{u t}=0$ and $\epsilon(k)=0.5 * \epsilon(k)$ and go to step 6. If $\epsilon(k) \leq \epsilon_{m}$, then keep $\epsilon(k)$ unaltered and go to step 6 .

6) If $k$ lies in the given run time $t$, go to step 2; otherwise record the outputs of each array instants and stop.

Remark 5: The proposed ETM (10) also takes into account both the error dynamics and control traffic, as discussed above. The low-gain parameter $\epsilon(k)$ is dynamically adjusted as per the network load and can achieve optimal utilization of the network resources while the parameter $\sigma(k)$ is adjusted according to the error dynamics. Therefore, we can achieve satisfactory performance without causing heavy traffic or network congestion.

\section{Inter-event time}

This section shows the effect of $\epsilon$ on the inter-event time. Before proceeding to the main Theorem, a Lemma is provided, which will be used in the subsequent proofs.

Lemma 4: [42] Consider a square matrix $A$, there exist positive scalars $\rho(A), \mathbf{M}$, and $\Delta$ such that for each non negative power $k$, it satisfies

$$
\|A\|^{k} \leq M(\rho(A)+\Delta)^{k}
$$

where $\Delta>0, M \geq 1$, and $\rho(A)$ is the spectral radius of the given matrix $A$.

For the subsequent analysis we define the design parameters $M=1, \Delta \leq 0.001$. Event-triggered parameters are considered as $\beta>2, d_{0}<1,0.5 \leq d_{1}<1$. Initial condition of dynamic variable is considered as $\sigma(0)=1$. Furthermore, the proof is carried out by assuming matrix $A$ to be nonzero.

Theorem 2: For the event-triggered system (26b) and the triggering rule (10), the sequence of triggering instants $\{q, q+1, \ldots\}$ ensures that $\forall k>0$, the interval between any two consecutive events is given by

$$
\tau \geq\left\lfloor n_{j}\right\rfloor T_{s}
$$

where,

$$
\begin{aligned}
& n_{j} \geq \frac{1}{\ln \delta}\left[\ln \left(\bar{\sigma}\left(d_{0}+\beta d_{1}^{k+n_{j}}\right) \frac{\delta-1}{\gamma}+1\right)-\ln (\delta)\right]+1 \\
& \delta:=M(\rho(A)+\Delta) \\
& \gamma:=\|u(q+j-1)\|(I+\|G\|\|B\|+M(\rho(A)+\Delta))
\end{aligned}
$$

$T_{s}$ is the sampling period. $n_{j}$ represents the number of periodic instants between triggering instants $(q+j-1, q+j)$ for all $j \geq 1$ and $\left\lfloor n_{j}\right\rfloor$ represents the nearest integer value less than or equal to $n_{j}$.

Proof: Consider current event is triggered at $k=(q+j-1)^{t h}$ instant, error for the next periodic instant is written as

$e_{u}(k+1)=u(q+j-1)-u(k+1), \forall k \in(q+j-1, q+j]$

Taking norms on both sides, we have

$$
\left\|e_{u}(k+1)\right\| \leq\|u(q+j-1)\|+\|G x(k+1)\|
$$

Expanding $x(k+1)$ in (54), norm of error is bounded by

$$
\begin{aligned}
\left\|e_{u}(k+1)\right\| & \leq\|u(q+j-1)\| \\
& +\|G A x(k)+G B u(q+j-1)\| \\
\left\|e_{u}(k+1)\right\| & \leq\|u(q+j-1)\|(I+\|G\|\|B\|) \\
& +\|G A x(k)\|
\end{aligned}
$$




$$
\begin{aligned}
\left\|e_{u}(k+1)\right\| & \leq\|u(q+j-1)\|(I+\|G\|\|B\|) \\
& +\left\|\lambda_{\max }(A)\right\|\|G x(k)\|
\end{aligned}
$$

Using Lemma 4, $\left\|e_{u}(k+1)\right\|$ in (57) is further bounded as

$$
\begin{gathered}
\left\|e_{u}(k+1)\right\| \leq\|u(q+j-1)\|(I+\|G\|\|B\|) \\
+M(\rho(A)+\Delta)\|G x(k)\| \\
\left\|e_{u}(k+1)\right\| \leq\|u(q+j-1)\|(I+\|G\|\|B\|+M(\rho(A)+\Delta)) \\
-M(\rho(A)+\Delta)\left\|e_{u}(k)\right\|
\end{gathered}
$$

$u(q+j-1)$ remains unaltered until the next event is triggered. Now using (52a) and (52b), the above inequality reduces to

$$
\left\|e_{u}(k+1)\right\| \leq \gamma+\delta\left\|e_{u}(k)\right\|
$$

Solving (60), we obtain

$$
\left\|e_{u}(k)\right\| \leq \sum_{s=0}^{n_{j}-1}\left(\delta^{n_{j}-1-s} \gamma\right), k \in[q+j-1, q+j)
$$

with $e_{u}(q+j-1)=0$. After simplification, $\left\|e_{u}(k)\right\|$ in (61) is bounded as

$$
\left\|e_{u}(k)\right\| \leq \frac{\delta^{n_{j}-1} \delta-1}{\delta-1} \gamma
$$

where, $n_{j}$ is the number of discrete periodic samples between two consecutive events $(q+j-1)^{\text {th }}$ event and $(q+j)^{\text {th }}$ event for all $j \geq 1$. Combining (62) and ETM (10), and considering the next event is triggered at $k=(q+j)^{t h}$ instant, we obtain

$$
\frac{\delta^{n_{j}-1} \delta-1}{\delta-1} \gamma \geq \sigma(k)\left(d_{0}+\beta d_{1}^{q+j}\right)
$$

Using (21), (63) can be further written as

$$
\frac{\delta^{n_{j}-1} \delta-1}{\delta-1} \gamma \geq \bar{\sigma}\left(d_{0}+\beta d_{1}^{q+j}\right)
$$

where, $q+j:=\left(k+n_{j}\right)^{t h}$ instant.

$$
\begin{aligned}
& \delta^{n_{j}-1} \delta \geq \bar{\sigma}\left(d_{0}+\beta d_{1}^{k+n_{j}}\right) \frac{\delta-1}{\gamma}+1 \\
& \ln \left(\delta^{n_{j}-1} \delta\right) \geq \ln \left(\bar{\sigma}\left(d_{0}+\beta d_{1}^{k+n_{j}}\right) \frac{\delta-1}{\gamma}+1\right)
\end{aligned}
$$

After simplification we obtain

$$
n_{j}-1 \geq \frac{1}{\ln (\delta)}\left[\ln \left(\bar{\sigma}\left(d_{0}+\beta d_{1}^{k+n_{j}}\right) \frac{\delta-1}{\gamma}+1\right)-\ln (\delta)\right]
$$

Thus, number of discrete instants $n_{j}$ between any two consecutive events is given by

$$
n_{j} \geq \frac{1}{\ln (\delta)}\left[\ln \left(\bar{\sigma}\left(d_{0}+\beta d_{1}^{k+n_{j}}\right) \frac{\delta-1}{\gamma}+1\right)-\ln (\delta)\right]+1
$$

In (67), right hand side may be a non integer. However, the nearest integer of $n_{j}$ satisfying (67) to be used as $n_{j}:=\left\lfloor n_{j}\right\rfloor$.

Hence, inter-event time is bounded as $\tau \geq\left\lfloor n_{j}\right\rfloor T_{s}$. This completes the proof.
Remark 6: In the case of periodic controller, system measurements are transmitted at every periodic instant i.e. it takes exactly one time step to transmit the system measurements; while using ETM, system measurements are not transmitted at every time steps, which implies the ETM takes at least one time step to trigger an event. Noticing that, for the given values of design parameters, $\bar{\sigma}\left(d_{0}+\beta d_{1}^{k+n_{j}}\right) \frac{\delta-1}{\delta}+1 \geq \delta$, which results in $\frac{1}{\ln \delta} \ln \left[\bar{\sigma}\left(d_{0}+\beta d_{1}^{k+n_{j}}\right) \frac{\delta-1}{\delta}+1-\ln (\delta)\right]>0$ and $n_{j}>1$ in (67).

Theorem 3: Consider the system (26b) with feedback gain designed using (4) and triggering rule (10). Then the interevent time is monotonically increasing with decrease in $\epsilon$.

Proof: Consider $\epsilon_{1} \in \epsilon, \epsilon_{2} \in \epsilon$ such that $\epsilon_{1}<\epsilon_{2}$. It should be noted that all the parameters except $\gamma$ in (67) are independent of $\epsilon$. Now evaluating the ratio between $n_{j}$ for both $\epsilon_{1}$ and $\epsilon_{2}$ as

$$
\frac{\left.n_{j}\right|_{\epsilon_{1}}}{\left.n_{j}\right|_{\epsilon_{2}}}=\frac{\frac{1}{\ln \delta}\left[\ln \left(\bar{\sigma}\left(d_{0}+\beta d_{1}^{k+n_{j}}\right) \frac{\delta-1}{\gamma_{1}}+1\right)-\ln (\delta)\right]+1}{\frac{1}{\ln \delta}\left[\ln \left(\bar{\sigma}\left(d_{0}+\beta d_{1}^{k+n_{j}}\right) \frac{\delta-1}{\gamma_{2}}+1\right)-\ln (\delta)\right]+1}
$$

It can be observed that only $\gamma_{1}, \gamma_{2}$ are dependent on $\epsilon$ and

$$
\gamma_{1}<\gamma_{2}, \forall \epsilon_{1}<\epsilon_{2}
$$

Hence, $\frac{1}{\gamma_{1}}>\frac{1}{\gamma_{2}}, \forall \epsilon_{1}<\epsilon_{2}$ will hold. which results in

$$
\bar{\sigma}\left(d_{0}+\beta d_{1}^{k+n_{j}}\right) \frac{\delta-1}{\gamma_{1}}>\bar{\sigma}\left(d_{0}+\beta d_{1}^{k+n_{j}}\right) \frac{\delta-1}{\gamma_{2}}
$$

Except $\gamma_{1}, \gamma_{2}$, all the parameters in (70) are fixed positive scalars and by design $\bar{\sigma}\left(d_{0}+\beta d_{1}^{k+n_{j}}\right) \frac{\delta-1}{\gamma_{i}}>\delta>1, i=\{1,2\}$. This implies

$$
\begin{aligned}
& \ln \left(\bar{\sigma}\left(d_{0}+\beta d_{1}^{k+n_{j}}\right) \frac{\delta-1}{\gamma_{1}}+1\right)-\ln (\delta)> \\
& \ln \left(\bar{\sigma}\left(d_{0}+\beta d_{1}^{k+n_{j}}\right) \frac{\delta-1}{\gamma_{2}}+1\right)-\ln (\delta)
\end{aligned}
$$

which leads to

$$
\frac{\frac{1}{\ln \delta}\left[\ln \left(\bar{\sigma}\left(d_{0}+\beta d_{1}^{k+n_{j}}\right) \frac{\delta-1}{\gamma_{1}}+1\right)-\ln (\delta)\right]+1}{\frac{1}{\ln \delta}\left[\ln \left(\bar{\sigma}\left(d_{0}+\beta d_{1}^{k+n_{j}}\right) \frac{\delta-1}{\gamma_{2}}+1\right)-\ln (\delta)\right]+1}>1
$$

i.e.

$$
\left.n_{j}\right|_{\epsilon_{1}}>\left.n_{j}\right|_{\epsilon_{2}}
$$

which implies that the number of discrete instants is increased when $\epsilon$ is decreased. This completes the proof.

Remark 7: This monotonic decaying nature of feedback gain can significantly reduce the control traffic at any particular instant by extending the inter-event time at that instant depending on the traffic condition and event density as discussed in the Algorithm. 
TABLE I

PHYSICAL PARAMETERS OF A QUARTER CAR ACTIVE SUSPENSION MODEL

\begin{tabular}{|l|l|l|l|}
\hline symbol & value & symbol & value \\
\hline$k_{s}$ & $900 \mathrm{~N} / \mathrm{m}$ & $k_{u s}$ & $2000 \mathrm{~N} / \mathrm{m}$ \\
$B_{s}$ & $7.5 \mathrm{Ns} / \mathrm{m}$ & $B_{u s}$ & $5 \mathrm{Ns} / \mathrm{m}$ \\
$M_{s}$ & $2.5 \mathrm{~kg}$ & $M_{u s}$ & $1 \mathrm{~kg}$ \\
\hline
\end{tabular}

\section{Simulation RESUlTS}

Example In this example, a practical quarter car active suspension model [43] is considered to illustrate the advantages and effectiveness of the proposed DETM under various road disturbances. The state space model of equations of motion of the active suspension model can written as $\dot{x}(t)=$ $A_{c} x(t)+B_{c} F_{c}(t)+E_{c} \dot{z}_{r}(t) \cdot x(t)=\left[\begin{array}{llll}x_{1} & x_{2} & x_{3} & x_{4}\end{array}\right]^{T}=$ $\left[\begin{array}{llll}z_{s}-z_{u} & z_{u}-z_{r} & \dot{z}_{s} & \dot{z}_{r}\end{array}\right]^{T} \cdot z_{s}-z_{u}$ represents the suspension deflection, $\dot{z}_{s}$ represents the velocity of the vehicle body, $z_{u}-z_{r}$ represents the road-tire deflection and $\dot{z}_{r}$ indicates the velocity of the road surface. $\ddot{z}_{s}$ is the vertical acceleration, which is also represented as $z_{v}$ in simulation results. The matrices $A_{c}, B_{c}, E_{c}$ are given as

$$
\begin{gathered}
A_{c}=\left[\begin{array}{cccc}
0 & 0 & 1 & -1 \\
0 & 0 & 0 & 1 \\
\frac{-k_{s}}{m_{s}} & 0 & \frac{-B_{s}}{M_{s}} & \frac{B_{s}}{M_{s}} \\
\frac{k_{u s}}{M_{u s}} & \frac{-k_{u s}}{M_{u s}} & \frac{B_{s}}{M_{u s}} & \frac{-B_{s}+B_{u s}}{M_{u s}}
\end{array}\right] \\
B_{c}=\left[\begin{array}{llllll}
0 & 0 & \frac{1}{M_{s}} & \frac{-1}{M_{u s}}
\end{array}\right]^{T}, E_{c}=\left[\begin{array}{llll}
0 & -1 & 0 & \frac{B_{u s}}{M_{u s}}
\end{array}\right]^{T}
\end{gathered}
$$

$M_{s}$ and $M_{u s}$ represent the vehicle body mass and mass of the tyre, respectively. $k_{s}$ represents spring stiffness between car body and tyre, $k_{u s}$ denotes spring stiffness between tyre and road. $B_{s}$ and $B_{u s}$ are damping coefficient between car body and tyre, and damping coefficient between tyre and road, respectively.

In order to design the controller, system is first discretized and the equivalent discrete-time system model with sampling period $T_{s}=0.08 \mathrm{~s}$ is given by

$$
x(k+1)=A x(k)+B F_{c}(k)+E z_{r}(k)
$$

where, $A=e^{A_{c} T_{s}}, B=\int_{0}^{T_{s}} e^{A_{c} t} B_{c} d t, E=\int_{0}^{T_{s}} e^{A_{c} t} E_{c} d t$. $x=\left[\begin{array}{llll}x_{1} & x_{2} & x_{3} & x_{4}\end{array}\right]^{T}$ is the state vector. $F_{c}(k)$ denotes the input force, which is applied between the wheel and vehicle body. This input force is the active component of the suspension system. Another input to the system is the derivative of road surface position $z_{r}(k)$. All the open-loop eigenvalues of discretized system matrix $A$ are given by $(0.5861+$ $0.7587 i, 0.5861-0.7587 i, 0.9879+0.1406 i, 0.9879-0.1406 i)$, respectively. In an active-suspension system, the road profile is usually interpreted as an external disturbance. Using the lowgain concept, the feedback gain is computed using the eigenstructure assignment method with closed-loop eigenvalues $\lambda_{c}$. $\lambda_{c}=(1-\epsilon) \lambda_{o}$, where $\lambda_{o}$ denotes the open-loop eigenvalues of system matrix $A$.

Numerical simulation: The simulations are used to verify the efficacy of the designed control paradigm. We examine the following ETMs to compare the results.

1) In SETM, the updated control input is sent to the actuator through a network in an event-triggered manner.
However, the threshold parameters, including low-gain variable $(\epsilon)$ are permanently fixed. The static ETM can be realized by setting $\sigma(0)>0$ and $c_{3}=\theta=0$. For the given example $\sigma(0)=0.1, c_{1}=1, \bar{\theta}=0.1$ and $c_{2}=0.01$ in (11). SETM is performed for the fixed low-gain parameter $\epsilon=0.05$ and 0.1 to show the effect of $\epsilon$ on the system performance.

To further compare the results, we consider SETM proposed in [29] and the input error-based ETM (see subsection 3.2 in [44]). For fair comparison, low-gain parameter is fixed at $\epsilon=0.05$.

2) In the case of DETM, triggering an event depends on error dynamics and event density. The triggering threshold is dynamically adjusted according to the dynamic rule, and the low-gain parameter is adjusted as per event density, as described in the Algorithm. For the proposed DETM, the design parameters are chosen as $d_{0}=0.01, d_{1}=0.8, \beta=2, \sigma(0)=1, \bar{\theta}=0.0006, \theta=$ 0.0004 . To show the effect of the adaptive $\epsilon$ on network load, the simulation is performed for both fixed $\epsilon$ and adaptive $\epsilon$.

To compare the results, we consider DETM proposed in [29]. The similar structure of DETM is widely used in many works.

3) In this case, system measurements are transmitted and control input is updated at each discrete instant. By enforcing $\sigma(0)=0$ and $c_{1}=c_{3}=c_{2}=0$ in triggering rule (10) and dynamic law (11), the controller becomes periodic one.

In the subsequent simulation results, the run time and sampling period were set as $50 \mathrm{sec}$ and $0.08 \mathrm{sec}$, respectively. For the given run time and sampling period, the total number of samples 625. To illustrate the effectiveness of the proposed ETM, fundamental issues such as ride comfort, road disturbance and suspension deflection of the given active suspension for different road profiles are taken into account as the performance criteria. Furthermore, these road profiles are considered as road disturbance, which can either be classified as bump response (road profile I) or random signal (road profile II). Root-mean square (RMS) values are used for quantitative analysis. It is noted that ride comfort can be quantified by the body's vertical acceleration. Road holding of the vehicle has an effective role during the manoeuvres such as accelerating, braking, etc. In the following, the road profiles are used to validate the performance of the proposed control approach as well as the effect of low-gain parameter on the performance for both the static/dynamic ETMs, as discussed above.

Performance analysis for different Road profiles (R. P.): Road profile 1 is considered as the rectangular pulse with $5 \mathrm{~mm}$ height and $10 \%$ duty cycle and road profile 2 is considered as random signal with $5 \mathrm{~mm}$ height.

In the periodic control paradigm, the system outputs for the suspension system are depicted in Fig. 5. As, control input is updated at every instants; therefore, number of events is maximum (625 for the given run-time), which may increase the network load and cause delay and network traffic. However, 
system performance may further worsened with increase in the irregularities in road dynamics.

It can be observed from Fig. 3 that ride comfort is improved with an increase in $\epsilon$ for both the road profiles. However, the number of events is also increased in case of high $\epsilon$, which will cause network congestion and network traffic; thus, for network resource-constrained suspension system, SETM with high $\epsilon$ is not very suitable. On the other hand, low values of $\epsilon$ cause fewer events but at the price of ride comfort, which can be observed from Fig. 3, Table II and Table III.

From Table II and Table III, it can be observed that the triggering mechanisms have better response in case of road profile I compared to the road profile II. The RMS values of the vertical deflection, suspension deflection and tyre deflection are decreased compared to the road profile II, which indicates that suspension performance (including ride comfort, road handling) is improved for bump response. Second, in case of static ETMs (state-dependent [29], input-dependent proposed ETM), system response has been degraded vis-a-vis corresponding DETM, this has been observed from the Table II- Table IV where RMS values is less for DETM. For the fair comparison, we keep $\epsilon=0.05$. Further, number of events are less in the proposed DETM compared to the static ETM, as depicted in Fig. 3d and Fig. 4e.

Furthermore, a quantitative comparison of RMS values are provided in Table II- Table IV for different control paradigms. It can also be observed that (1) Compared to the proposed static input-based ETM, proposed DETM (fixed and adaptive $\epsilon)$ significantly reduces the sprung mass acceleration in the presence of road disturbance. (2) Reduces the tyre and road deflection and vehicle suspension deflection. (3) RMS values of the proposed DETM is less compared to the proposed DETM in [29], which indicates that the proposed DETM outperforms the DETM [29]. It should be noted that, most of the dynamic ETMs are designed using the similar structure as in [29]. It is found that inter-event time is extended adaptively at any particular instant by decreasing $\epsilon$, while may not be possible in the case of state-based DETM in [29], which can be observed from Fig. 4e (adaptive $\epsilon$ based DETM). (4) Number of events is also less in the proposed DETM (for adaptive $\epsilon$ ) compared to the periodic controller, DETM [29], and static ETMs [29], [44] and proposed input error-based static ETM, which implies that the proposed DETM requires less resources vis-a-vis the other triggering mechanisms, as mentioned above.

From the simulation results, it can be concluded that

1) The constrained and network resources are efficiently utilized using the proposed DETM while maintaining a satisfactory ride performance. On the other hand, optimal utilization of network resources can be achieved while degrading a small amount of ride performance using the DETM proposed in [29]. The proposed DETM has a larger possibility compared to the DETM considered in [29] due to the introduction of adaptive gain. Through the dynamic ETM, we can increase the feedback gain when the vertical acceleration suffers from more oscillations, and the road surface is not smooth. On the other hand, we can decrease the feedback to reduce the control execution in the networked control en- vironment. Thus, the proposed DETM can significantly reduce the number of updates and data transmission and provides a better trade-off between suspension control performance and communication efficiency vis-a-vis the periodic and static counterparts.

2) As the design parameters in the proposed static ETM is independent of change in dynamics; therefore, the event number may not be equal to the required number of events, which can degrade the system's performance. This can be also be shown in Table II (for fixed $\epsilon=0.05)$.

3) With fixed $\epsilon$, the number of events is always less in static ETM vis-a-vis proposed DETM (see Table II, Table III). It may be for the following reason: $\sigma(k)$ is fixed in STEM, but in DETM, $\sigma(k)$ is monotonically decreasing. Therefore the event-triggered threshold is comparatively less in DETM. However, this problem has been solved in the proposed DETM by using the adaptive value of $\epsilon$ instead of its fixed value, as shown in Fig. 3d and Fig. 4e. Furthermore, number of events for input-based ETM [44] is less than the proposed static ETM for the given value of $\epsilon$, as shown in Fig. 3d and Fig. 6c. However, the proposed ETM has an additional advantage due to the presence of $\epsilon$. By increasing $\epsilon$, system performance can be improved.

\section{CONCLUSION}

In this paper, dynamic input-based ETM with a low-gain approach has been investigated. The parameterized $(\epsilon)$ controller with the proposed ETM permits delaying the next event at any particular instant; this is particularly useful in a resourceconstrained environment. Also, due to input-based error, the control law is updated only when there is a significant change in input compared to the past updated input.

The relationship between $\epsilon$ and inter-event time has been established analytically, which shows that decrease in $\epsilon$ increases the inter-event time at a particular instant which has been validated in simulation results. Stability has been established using ISS Lyapunov function. Finally, simulation results have been exploited to illustrate the advantages and effectiveness of the proposed study. Comparison between state-dependent ETM and input-dependent ETM has been presented in the simulation. Also, the comparative results of the periodic sampling mechanism, the static ETM with a constant low-gain parameter $\epsilon$, have been provided to demonstrate the superiority of the proposed dynamic ETM in the result section. The proposed work has significantly reduced redundant data transmissions over the network, as shown above. It has been indicated from the simulation that the proposed controller can achieve satisfactory ride comfort in the case of quarter car active suspension while saving more network resources at the same time. The proposed methodology has shown its promising performance for a network resource-constrained system in simulations.

\section{REFERENCES}

[1] K. J. Astrom and B. M. Bernhardsson, "Comparison of riemann and lebesgue sampling for first order stochastic systems," in Proceedings of the 41st IEEE Conference on Decision and Control, 2002., vol. 2. IEEE, 2002, pp. 2011-2016. 
TABLE II

RMS VALUES OF THE SUSPENSION CHARACTERISTICS FOR THE PROPOSED ETMS AND PERIODIC CONTROL WITH ROAD PROFILE I

\begin{tabular}{|l|l|l|l|l|l|}
\hline Parameters & $\begin{array}{l}\text { Periodic } \\
\text { controller }\end{array}$ & $\begin{array}{l}\text { SETM } \\
(\text { low } \epsilon \text { ) }\end{array}$ & $\begin{array}{l}\text { DETM } \\
\text { (fixed } \epsilon \text { ) }\end{array}$ & $\begin{array}{l}\text { SETM } \\
\text { (high } \epsilon \text { ) }\end{array}$ & $\begin{array}{l}\text { DETM } \\
\text { adaptive } \\
\epsilon \text { ) }\end{array}$ \\
\hline $\begin{array}{l}\text { vertical ac- } \\
\text { celeration } \\
\text { Suspension } \\
\text { deflection } \\
\text { Tyre deflec- } \\
\text { tion }\end{array}$ & 0.0009 & 0.0057 & 0.0026 & 0.0144 & 0.0061 \\
\hline
\end{tabular}

TABLE III

RMS VALUES OF THE SUSPENSION CHARACTERISTICS FOR THE PROPOSED ETMS AND PERIODIC CONTROL WITH ROAD PROFILE II

\begin{tabular}{|l|l|l|l|l|l|}
\hline Parameters & $\begin{array}{l}\text { Periodic } \\
\text { controller }\end{array}$ & $\begin{array}{l}\text { SETM } \\
\text { (low } \epsilon)\end{array}$ & $\begin{array}{l}\text { DETM } \\
\text { (fixed } \epsilon \text { ) }\end{array}$ & $\begin{array}{l}\text { SETM } \\
\text { (high } \epsilon \text { ) }\end{array}$ & $\begin{array}{l}\text { DETM } \\
\text { (adaptive } \\
\epsilon\end{array}$ \\
\hline $\begin{array}{l}\text { vertical ac- } \\
\text { celeration } \\
\text { Suspension } \\
\text { deflection } \\
\text { Tyre deflec- } \\
\text { tion }\end{array}$ & 0.0321 & 0.0357 & 0.025 & 0.0194 & 0.0108 \\
\hline
\end{tabular}

[2] P. Tabuada, "Event-triggered real-time scheduling of stabilizing control tasks," IEEE Transactions on Automatic Control, vol. 52, no. 9, pp. $1680-1685,2007$.

[3] J. Zhang, D. Zhao, and W. X. Zheng, "Output feedback control of discrete-time systems with self-triggered controllers," International Journal of Robust and Nonlinear Control, vol. 25, no. 18, pp. 36983713, 2015.

[4] Y. Wang, Z. Jia, and Z. Zuo, "Dynamic event-triggered and selftriggered output feedback control of networked switched linear systems," Neurocomputing, vol. 314, pp. 39-47, 2018.

[5] R. Yang and W. X. Zheng, "Output-based event-triggered predictive control for networked control systems," IEEE Transactions on Industrial Electronics, 2019.

[6] Y. Xu, M. Fang, Z.-G. Wu, Y.-J. Pan, M. Chadli, and T. Huang, "Inputbased event-triggering consensus of multiagent systems under denial-ofservice attacks," IEEE Transactions on Systems, Man, and Cybernetics. Systems, 2018

[7] A. Girard, "Dynamic triggering mechanisms for event-triggered control," IEEE Transactions on Automatic Control, vol. 60, no. 7, pp. 1992-1997, 2014.

[8] A. Sahoo, H. Xu, and S. Jagannathan, "Near optimal event-triggered control of nonlinear discrete-time systems using neurodynamic programming," IEEE transactions on neural networks and learning systems, vol. 27, no. 9, pp. 1801-1815, 2015.

[9] N. Zhou, R. Chen, Y. Xia, J. Huang, and G. Wen, "Neural networkbased reconfiguration control for spacecraft formation in obstacle environments," International Journal of Robust and Nonlinear Control, vol. 28, no. 6, pp. 2442-2456, 2018.

[10] X. Xie, Q. Zhou, D. Yue, and H. Li, "Relaxed control design of discrete-time takagi-sugeno fuzzy systems: An event-triggered realtime scheduling approach," IEEE Transactions on Systems, Man, and Cybernetics: Systems, vol. 48, no. 12, pp. 2251-2262, 2018.

[11] L. Zhang and M. Z. Chen, "Event-triggered control for semi-global

TABLE IV

RMS VALUES OF SUSPENSION CHARACTERISTICS FOR DIFFERENT THRESHOLD VALUES OF ETM [29], [44] AND PERIODIC CONTROL WITH ROAD PROFILES (R. P. I, R. P. II)

\begin{tabular}{|l|l|l|l|}
\hline Parameters & $\begin{array}{l}\text { SETM [44] (R.P. } \\
\text { I, R.P. II) }\end{array}$ & $\begin{array}{l}\text { SETM [29] (R.P. } \\
\text { I, R.P. II) }\end{array}$ & $\begin{array}{l}\text { DETM [29] (R.P. } \\
\text { I, R.P. II) }\end{array}$ \\
\hline $\begin{array}{l}\text { vertical ac- } \\
\text { celeration } \\
\begin{array}{l}\text { Suspension } \\
\text { deflection } \\
\text { Tyre deflec- } \\
\text { tion }\end{array}\end{array}$ & $0.0154,0.0204$ & $0.0583,0.31$ & $0.0002,0.0481$ \\
\hline
\end{tabular}
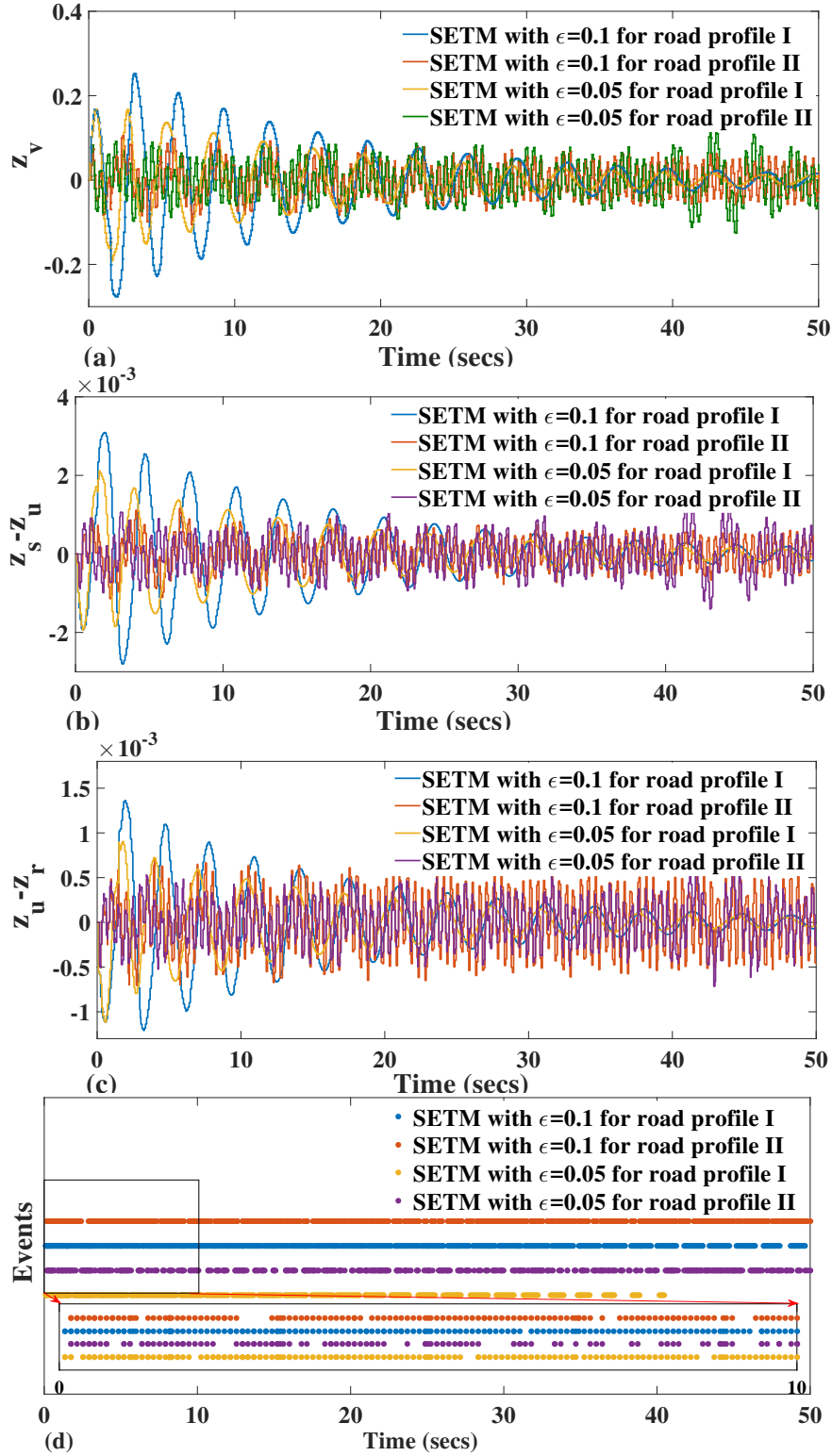

Fig. 3. a.Vertical acceleration b.Suspension deflection c. Tire deflection d. Events for static ETM for different road profiles and $\epsilon$

stabilisation of systems with actuator saturation," International Journal of Control, vol. 89, no. 5, pp. 1047-1064, 2016.

[12] D. Theodosis and D. V. Dimarogonas, "Event-triggered control of nonlinear systems with updating threshold," IEEE Control Systems Letters, vol. 3, no. 3, pp. 655-660, 2019.

[13] Y.-C. Sun and G.-H. Yang, "Periodic event-triggered resilient control for cyber-physical systems under denial-of-service attacks," Journal of the Franklin Institute, vol. 355, no. 13, pp. 5613-5631, 2018.

[14] H. Ren, G. Zong, and T. Li, "Event-triggered finite-time control for networked switched linear systems with asynchronous switching," IEEE Transactions on Systems, Man, and Cybernetics: Systems, vol. 48, no. 11 , pp. $1874-1884,2018$

[15] B. A. Khashooei, D. J. Antunes, and W. Heemels, "A consistent threshold-based policy for event-triggered control," IEEE Control Systems Letters, vol. 2, no. 3, pp. 447-452, 2018.

[16] M. Kishida, "Event-triggered control with self-triggered sampling for discrete-time uncertain systems," IEEE Transactions on Automatic Control, vol. 64, no. 3, pp. 1273-1279, 2018.

[17] T. Zhou, Z. Zuo, and Y. Wang, "Self-triggered and event-triggered control for linear systems with quantization," IEEE Transactions on 

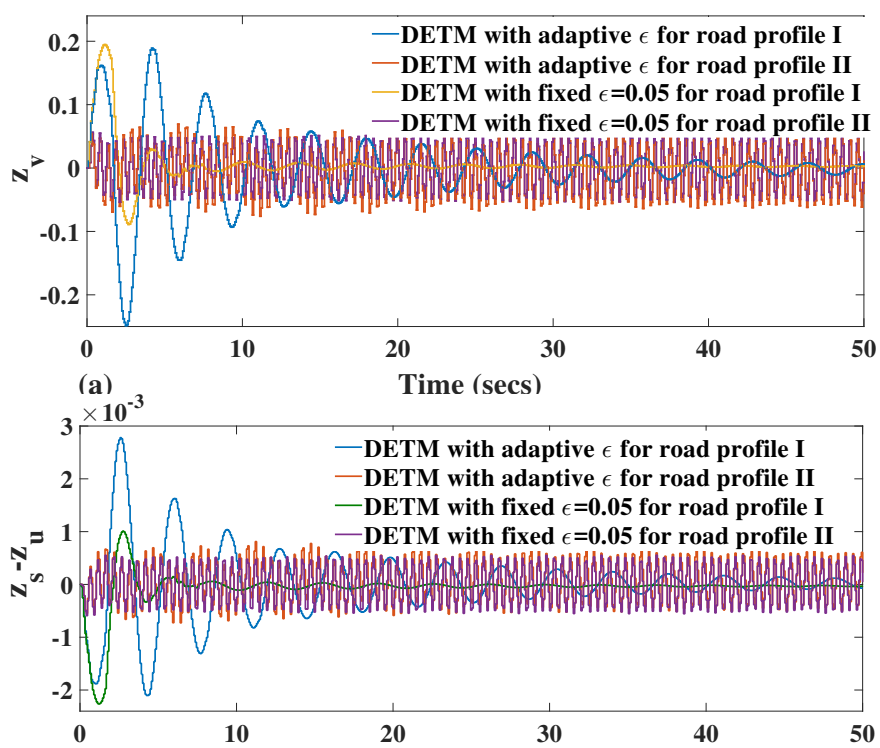

(b)
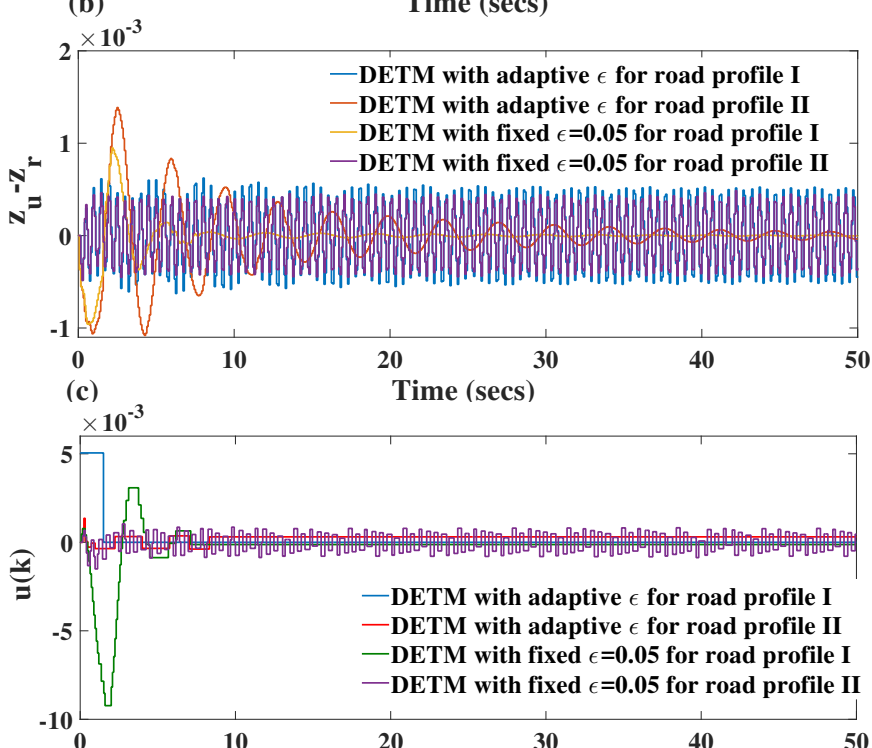

(d)

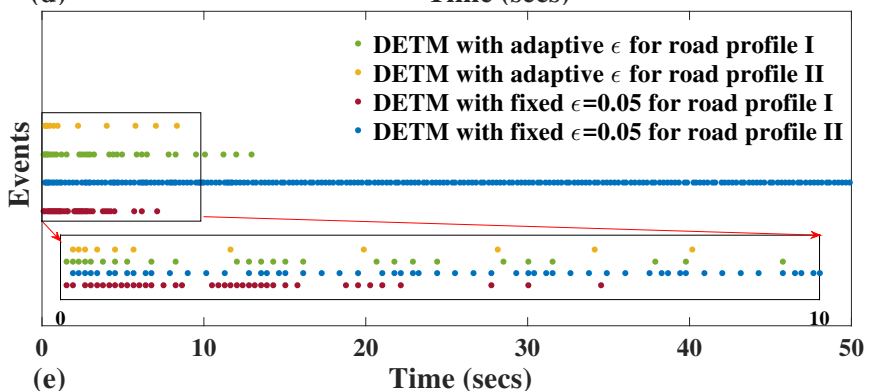

Fig. 4. Suspension characteristics for dynamic ETM for different road profiles and $\epsilon$

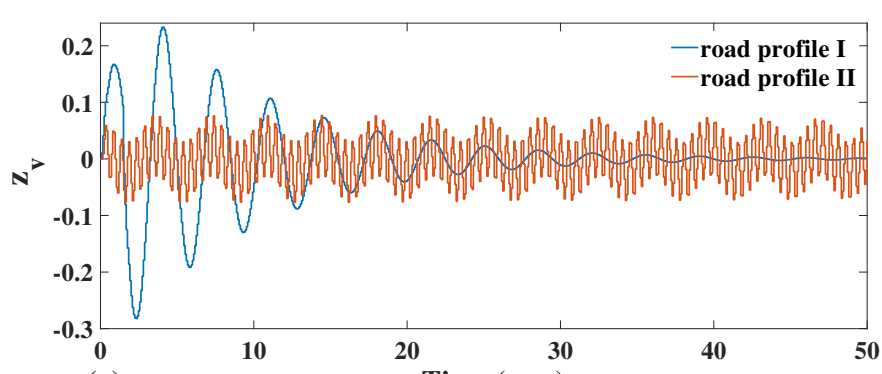

(a) Time (secs)

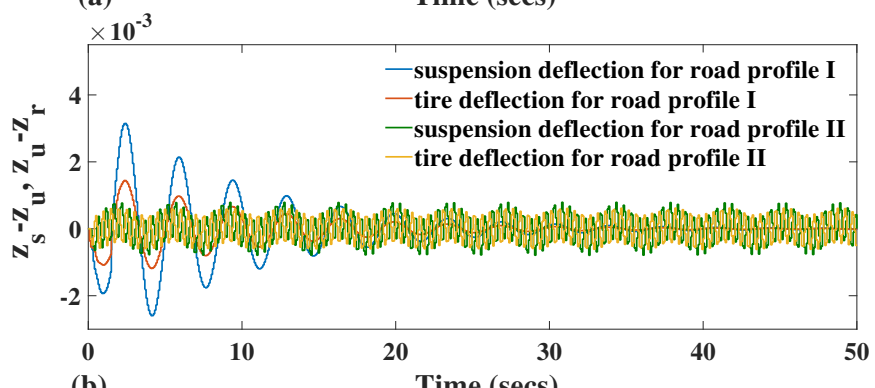

(b)

Time (secs)

Fig. 5. a.Vertical acceleration b.Suspension deflection and Tire deflection for periodic controller under different road profiles with $\epsilon=0.05$

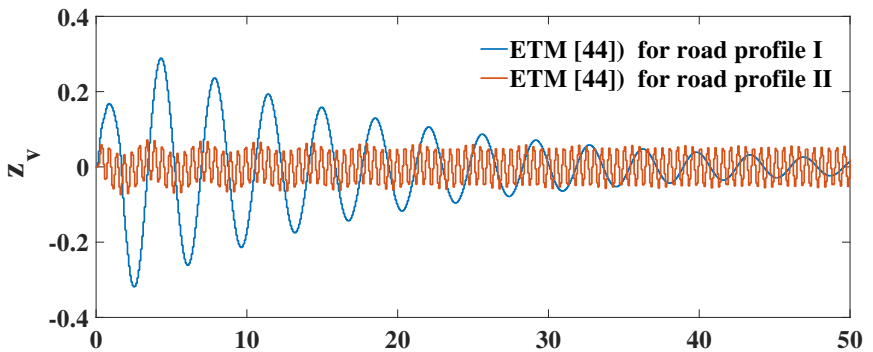

(a)
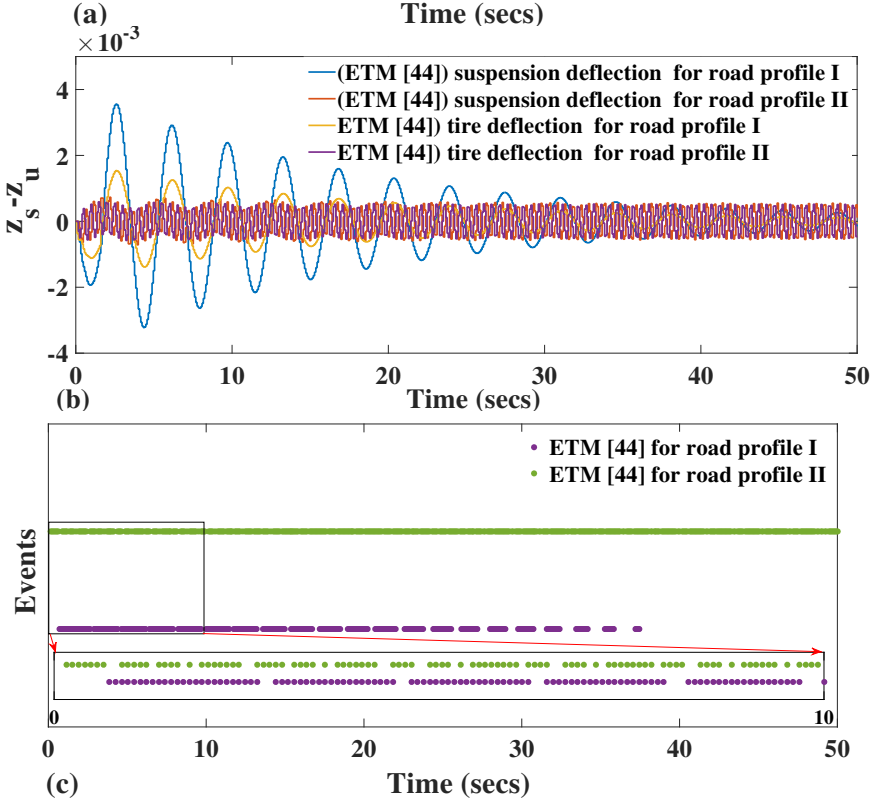

Fig. 6. Suspension characteristics for static ETM [44] for different road profiles and $\epsilon=0.05$ 


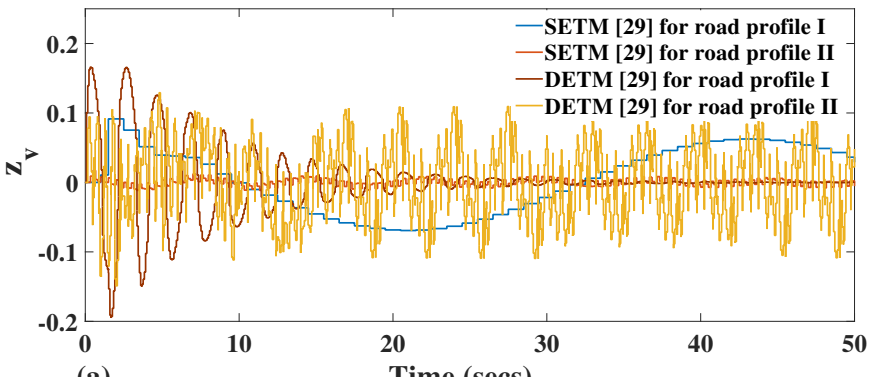

(a)

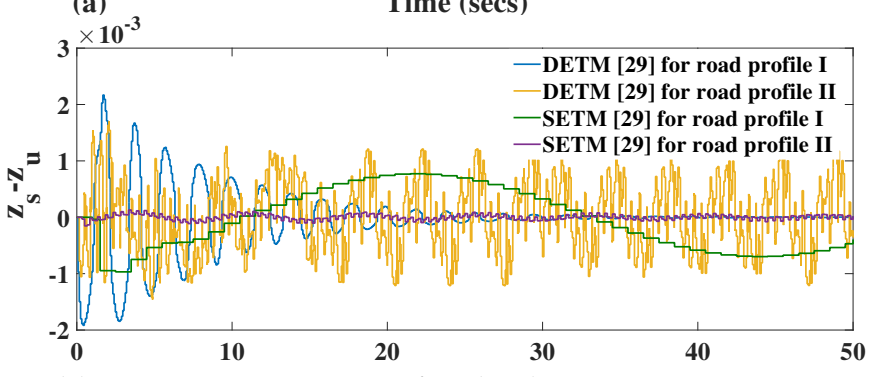

(b)
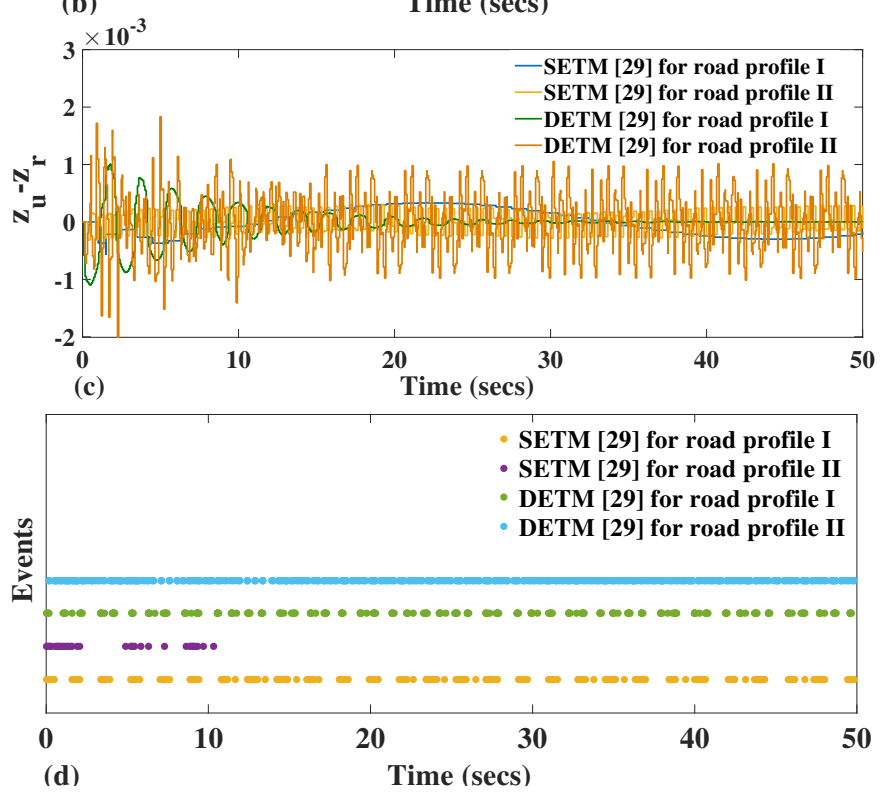

Fig. 7. Suspension characteristics for static ETM [29] for different road profiles and $\epsilon=0.05$

Systems, Man, and Cybernetics: Systems, 2018.

[18] X. Wang, H. Su, X. Wang, and G. Chen, "Fully distributed eventtriggered semiglobal consensus of multi-agent systems with input saturation," IEEE Transactions on Industrial Electronics, vol. 64, no. 6, pp. 5055-5064, 2016.

[19] J. Liu, T. Yin, J. Cao, D. Yue, and H. R. Karimi, "Security control for ts fuzzy systems with adaptive event-triggered mechanism and multiple cyber-attacks," IEEE Transactions on Systems, Man, and Cybernetics: Systems, 2020.

[20] Y. Zhang, H. Li, J. Sun, and W. He, "Cooperative adaptive eventtriggered control for multiagent systems with actuator failures," IEEE Transactions on Systems, Man, and Cybernetics: Systems, vol. 49, no. 9, pp. 1759-1768, 2018.

[21] K. Zhang, B. Zhou, and H. Jiang, "Parametric lyapunov equation based event-triggered and self-triggered control of input constrained linear systems," International Journal of Robust and Nonlinear Control, 2019.

[22] X. Ge, Q.-L. Han, L. Ding, Y.-L. Wang, and X.-M. Zhang, "Dynamic event-triggered distributed coordination control and its applications: A survey of trends and techniques," IEEE Transactions on Systems, Man, and Cybernetics: Systems, vol. 50, no. 9, pp. 3112-3125, 2020.

[23] D. Ding, Z. Wang, and Q.-L. Han, "Neural-network-based consensus control for multiagent systems with input constraints: The event- triggered case," IEEE Transactions on Cybernetics, vol. 50, no. 8, pp. 3719-3730, 2020

[24] M. Guinaldo, D. Lehmann, J. Sanchez, S. Dormido, and K. H. Johansson, "Reducing communication and actuation in distributed control systems," in 52nd IEEE Conference on Decision and Control. IEEE, 2013, pp. 5288-5293.

[25] B. A. Khashooei, D. J. Antunes, and W. Heemels, "Output-based eventtriggered control with performance guarantees," IEEE Transactions on Automatic Control, vol. 62, no. 7, pp. 3646-3652, 2017.

[26] G. Wei, L. Liu, L. Wang, and D. Ding, "Event-triggered control for discrete-time systems with unknown nonlinearities: an interval observerbased approach," International Journal of Systems Science, vol. 51, no. 6, pp. 1019-1031, 2020.

[27] W. Chenliang, L. Yun, H. Qinglei, and J. Huang, "Event-triggered adaptive control for attitude tracking of spacecraft," Chinese Journal of Aeronautics, vol. 32, no. 2, pp. 454-462, 2019.

[28] L. Ma, Z. Wang, C. Cai, and F. E. Alsaadi, "A dynamic event-triggered approach to $\mathrm{h}$ control for discrete-time singularly perturbed systems with time-delays and sensor saturations," IEEE Transactions on Systems, Man, and Cybernetics: Systems, 2020.

[29] K. Zhang, B. Zhou, and G. Duan, "Event-triggered and self-triggered control of discrete-time systems with input constraints," IEEE Transactions on Systems, Man, and Cybernetics: Systems, 2020.

[30] S. Hajshirmohamadi, F. Sheikholeslam, M. Davoodi, and N. Meskin, "Event-triggered simultaneous fault detection and tracking control for multi-agent systems," International Journal of Control, vol. 92, no. 8, pp. 1928-1944, 2019.

[31] M. Ha, D. Wang, and D. Liu, "Event-triggered adaptive critic control design for discrete-time constrained nonlinear systems," IEEE Transactions on Systems, Man, and Cybernetics: Systems, vol. 50, no. 9, pp. 3158-3168, 2020.

[32] Y.-L. Wang, C.-C. Lim, and P. Shi, "Adaptively adjusted event-triggering mechanism on fault detection for networked control systems," IEEE Transactions on Cybernetics, vol. 47, no. 8, pp. 2299-2311, 2017.

[33] J. Sun, J. Yang, S. Li, and W. X. Zheng, "Estimate-based dynamic eventtriggered output feedback control of networked nonlinear uncertain systems," IEEE Transactions on Systems, Man, and Cybernetics: Systems, 2019.

[34] Y.-L. Wang, C.-C. Lim, and P. Shi, "Adaptively adjusted event-triggering mechanism on fault detection for networked control systems," IEEE Transactions on Cybernetics, vol. 47, no. 8, pp. 2299-2311, 2016.

[35] J. Zhang, C. Peng, D. Du, and M. Zheng, "Adaptive event-triggered communication scheme for networked control systems with randomly occurring nonlinearities and uncertainties," Neurocomputing, vol. 174, pp. 475-482, 2016.

[36] Z. Lin and Z. Lin, Low gain feedback. Springer, 1999.

[37] X. You, C. Hua, and X. Guan, "Event-triggered leader-following consensus for nonlinear multiagent systems subject to actuator saturation using dynamic output feedback method," IEEE Transactions on Automatic Control, vol. 63, no. 12, pp. 4391-4396, 2018.

[38] Z. Zuo, Q. Li, H. Li, and Y. Wang, "Event-triggered and self-triggered control for linear systems with actuator saturation," Transactions of the Institute of Measurement and Control, vol. 40, no. 4, pp. 1281-1288, 2018.

[39] Z.-P. Jiang and Y. Wang, "Input-to-state stability for discrete-time nonlinear systems," Automatica, vol. 37, no. 6, pp. 857-869, 2001.

[40] M. Guinaldo, D. V. Dimarogonas, K. H. Johansson, J. Sánchez, and S. Dormido, "Distributed event-based control strategies for interconnected linear systems," IET Control Theory \& Applications, vol. 7, no. 6, pp. 877-886, 2013.

[41] A. Eqtami, D. V. Dimarogonas, and K. J. Kyriakopoulos, "Eventtriggered control for discrete-time systems," in Proceedings of the 2010 American Control Conference, 2010, pp. 4719-4724.

[42] D. Dowler, "Bounding the norm of matrix powers," MS Thesis, Math. Dept., Brigham Young University, USA., 2013.

[43] S.-Y. Han, Y.-H. Chen, K. Ma, D. Wang, A. Abraham, and Z.-G. Liu, "Feedforward and feedback optimal vibration rejection for active suspension discrete-time systems under in-vehicle networks," in 2014 Sixth World Congress on Nature and Biologically Inspired Computing (NaBIC 2014). IEEE, 2014, pp. 139-144.

[44] W. Wu, S. Reimann, D. Görges, and S. Liu, "Event-triggered control for discrete-time linear systems subject to bounded disturbance," International Journal of robust and nonlinear control, vol. 26, no. 9, pp. 1902-1918, 2016. 